\title{
Erweiterte Röntgenkompetenz für MPA: Wo stehen wir?
}

\author{
M. Marchev, Präsident der kantonalen FMH-Delegierten für MPA-Fragen
}

Immer wieder wird mir die Frage gestellt, wieso die erweiterte Röntgenausbildung der MPA-Lehrtöchter noch immer nicht umgesetzt ist, das revidierte Reglement wurde doch bereits auf den 1. Dezember 2003 (!) in Kraft gesetzt.

Ich möchte im folgenden die aktuelle Sachlage und deren Entwicklung skizzieren.

\section{Situation vor der Revision (MPA-Ausbildungsreglement vom 12. September 1994)}

- Die MPA lernte, Extremitäten- und Thoraxröntgenbilder anzufertigen.

- Seit 15. September 1998 ist es für diplomierte MPA mit einem Jahr Berufserfahrung möglich, einen Weiterbildungskurs für das dosisintensive Röntgen zu absolvieren. Dieser Kurs wurde aber kaum besucht, da seine Bedingungen prohibitiv sind (Dauer, Kosten und vor allem die Anzahl zu testierender Aufnahmen innert eines Jahres [100 Aufnahmen bzw. sogar innert 3 Monaten 20 Schädelaufnahmen, was in den meisten Praxen nur mit bezüglich Strahlenschutz unethisch grosszügiger Indikationsstellung zu erreichen wäre]).

- 3947 Praxen (davon 2827 Grundversorger) haben aufgrund der Sachkunde des Praxisinhabers für dosisintensives Röntgen vom BAG eine entsprechende Röntgenberechtigung erhalten. Praktisch ausgeführt werden diese Aufnahmen seit je in den meisten Fällen von den MPA unter der Aufsicht des Berechtigten.

- In etlichen Schulen wurden schon seit einiger Zeit Grundlagen des dosisintensiven Röntgens vermittelt, um eine den praktischen Bedürfnissen angepasste Ausbildung zu gewährleisten.

\section{Revision des Ausbildungsreglements, in Kraft seit 1. Dezember 2003}

- Motivation für die Erweiterung der Röntgenkompetenz waren seitens der FMH und der MPA-Verbände folgende Überlegungen: a) Seit je werden in fast 4000 Praxen (davon fast 3000 Grundversorger) dosisintensive Aufnahmen unter Aufsicht des Sachkundeinhabers angefertigt.

b) Die letztlich ausführenden MPA hatten bisher keine praktikable Aus- oder Weiterbildungsmöglichkeit dazu.

c) Eine Verbesserung des Strahlenschutzes durch Integration der dosisintensiven Aufnahmen war das eigentliche Ziel dieses Teils der Ausbildungsrevision.

- Neu aufgenommen wurden die dosisintensiven Aufnahmen von Wirbelsäule, Becken, Abdomen und Schädel. Die theoretische Ausbildung wurde von 40 auf 70 Stunden aufgestockt. Die praktische Ausbildung umfasst nach wie vor 120 Stunden im Einführungskurs.

- Der Katalog der an der Lehrabschlussprüfung geprüften Aufnahmen wurde entsprechend angepasst.

- Diese Revision wurde vom BBT auf Antrag der FMH und der MPA-Verbände in Kraft gesetzt. Die Vernehmlassung war aus Zeitgründen kurz. Die Inkraftsetzung musste zwingend vor Inkrafttreten des neuen Berufsbildungsgesetzes (1. Januar 2004) stattfinden. Das BAG wurde über alle Schritte laufend orientiert.

\section{Das BAG blockiert auf Intervention der Radiologen, der MTRA (medizinisch- technische Röntgenassistentinnen und -assistenten) und der KSR (Kommission für Strahlenschutz und Radioaktivität) die Umsetzung des Reglements seit Anfang 2004}

Hier die wichtigsten Konfliktpunkte sowie die Gegenargumente der FMH:

- Radiologen und MTRA befürchten eine Verdrängung der MTRA durch die billigeren MPA mit erweiterter Röntgenkompetenz. Dies erscheint wenig stichhaltig, können die Radiologen doch schon heute MPA anstellen und sie in den seit 1998 bestehenden, von BAG und KSR homologierten Kurs schicken. 
Ausserdem umfasst das Röntgenberufsfeld der MTRA noch viele zusätzliche Gebiete (CT, MRI, US, Kontrastmitteluntersuchungen usw.).

- Die Umsetzung der praktischen Ausbildung wird Schwierigkeiten bereiten.

Dies ist sicher ein Problem, da auch heute die praktische Ausbildung in den konventionellen Aufnahmen in Lehrpraxen ohne Röntgen nicht einfach ist. Hier sind aber Modelle auf dem Tisch, die das Erreichen des vorgeschriebenen praktischen Katalogs während der ersten Berufsjahre ermöglichen. Dies wurde sowohl von BAG- als auch von Radiologenseite in einer Einigungskonferenz als praktikable Kompromissformel betrachtet.

- Eine Zunahme der dosisintensiven Aufnahmen wird befürchtet.

Dies ist unsinnig, da die Indikation von der Ärztin/dem Arzt gestellt wird, der die erforderliche Sachkompetenz haben muss. Richtig wäre allerdings, dass übertriebene Forderungen nach der Anzahl testierter Aufnahmen einen falschen Anreiz zur Ausweitung der Indikation stellen könnten. Aus diesem Grund wehren wir uns gegen eine unrealistische Zahl Pflichtaufnahmen.

- Die Anzahl der durchzuführenden Aufnahmen zur Erlangung einer genügenden Qualität der technisch anspruchsvolleren Aufnahmen des Achsenskeletts genüge nicht.

Auch eine suboptimale Ausbildung ist besser als keine. Die Aufnahmen werden bereits gemacht ... Auch bei den konventionellen Bildern können die MPA oft keine grosse Routine in der Lehre erlangen.

- Nur wenige MPA brauchen diese Zusatzausbildung.

Wie oben aufgeführt gibt es fast 4000 Träger der Sachkunde dosisintensives Röntgen, wovon fast 3000 als Grundversorger in der Praxis röntgen ...

\section{Einigungssitzung beim BBT am 13. Juli 2004, anwesend Vertreter von BBT, BAG, FMH (MPA-Verantwort- liche), MPA-Verbände, Radiologie und MTRA}

- Die Erweiterung der theoretischen Röntgenausbildung mit Einbezug der dosisintensiven Aufnahmen ist unbestritten.

- Die praktische Ausbildung wird von Radiologen- und MTRA-Seite als kaum umsetzbar erachtet, und es war nicht möglich, einen Konsens zu finden.

- Ebenso unmöglich war eine Einigung bezüglich Anforderungen für einen entsprechenden Ausweis.

\section{Im August 2004 liegt ein Kompromiss- vorschlag vor}

Er wurde bilateral von FMH und BAG erarbeitet, von FMH-Seite ausformuliert und dem BAG am 9. September 2004 offiziell als Antrag eingereicht, der Weg schien frei.

\section{Der Entscheid liegt seit unserer Eingabe vom 9. September 2004 bei BAG}

- Trotz mehrmaligem Nachfragen unsererseits hat das BAG bisher keinen verbindlichen Entscheid gefällt.

- Beim Treffen der zuständigen BAG-Vertreter mit den involvierten FMH-Entscheidungsträgern am 24. Januar 2005 zeichnet sich erneut eine Kompromisslösung ab.

- Beim jährlichen Treffen der kantonalen Röntgen-Chefexperten/-expertinnen im MPABereich am 27. Januar 2005 versicherte ein BAG-Vertreter, dass die Ausbildung im dosisintensiven Bereich in den Schulen wie geplant eingeführt werde und dass ein Kompromiss für die praktische Ausbildung in Griffweite sei.

- Anfang März 2005 fand ein Spitzentreffen FMH-Präsident und BAG-Direktor statt. Der BAG-Direktor sicherte einen baldigen Entscheid zu.

- Mitte März 2005 beschliesst die KSR (Kommission für Strahlenschutz und Überwachung der Radioaktivität) als beratendes Organ des BAG, die erweiterte Röntgenkompetenz der MPA abzulehnen. Dies, nachdem sich die Kommission in mehreren Sitzungen damit befasst hat. In der Begründung wird auf die meisten unserer Argumente gar nicht eingegangen, vgl. oben.

- Mitte August 2005 fehlt immer noch eine Antwort des BAG auf unseren im September 2004 eingereichten Vorschlag.

\section{Fazit einer unerträglichen Geschichte}

Zum jetzigen Zeitpunkt, rund 13/4 Jahre nach Inkrafttreten, tritt der erste nach neuem Reglement auszubildende Jahrgang ins 2. Lehrjahr 
ein. Schulen und Lehrmeister warten nach wie vor auf einen Entscheid des BAG, um endlich die Reglementsänderungen umsetzen zu können.

Der abschlägige Antrag der KSR ans BAG ist nicht nachvollziehbar und bringt keinerlei Verbesserung des Strahlenschutzes der Bevölkerung, da keine zusätzlichen Aufnahmen gemacht würden. Zudem: Der Anteil der Strahlenbelastung der Bevölkerung aus der radiologi- schen Diagnostik in den Grundversorgerpraxen beträgt nur gerade 9,5\%, wie eine vom BAG finanzierte Studie aus dem Institut für Röntgenphysik des KSR-Mitglieds Prof. J. F. Valley in Lausanne im Jahre 2000 nachgewiesen hat!

Dass sich das BAG durch eine beratende Kommission derart lähmen lässt und die Antwort auf einen vor einem Jahr gestellten, mit seinen Spezialisten vorbesprochenen Antrag schuldig bleibt, ist völlig unverständlich.

\section{Elargissement des compétences octroyées en radiologie aux assistantes médicales: état des lieux}

M. Marchev, président des délégués cantonaux de la FMH aux questions des assistantes médicales

L'on me demande régulièrement pourquoi l'élargissement des compétences octroyées en radiologie aux assistantes médicales n'est pas encore appliqué alors que le règlement révisé est entré en vigueur au $1^{\text {er }}$ décembre 2003 (!).

Je souhaite ci-après esquisser brièvement la situation actuelle et son évolution future.

\section{Situation avant la révision \\ (formation des assistantes médicales, règlement du 12 septembre 1994)}

- L'assistante médicale apprenait à effectuer les radiographies du thorax et des extrémités.

- Depuis le 15 septembre 1998, les assistantes médicales diplômées au bénéfice d'un an d'expérience professionnelle ont la possibilité de suivre un cours de perfectionnement en radiologie à fortes doses. Ce cours a cependant été peu fréquenté en raison des exigences prohibitives (durée, coûts et surtout nombre de clichés à effectuer en 1 an [100 clichés, dont 20 du crâne en 3 mois, ce qui, dans la plupart des cabinets médicaux, n'est possible qu'en posant des indications trop généreuses par rapport aux principes éthiques en matière de radioprotection]).
- 3947 cabinets médicaux (dont 2827 tenus par des médecins de premier recours) ont reçu une autorisation appropriée de l'OFSP en raison des qualifications techniques du détenteur du cabinet. Sur le plan pratique toutefois, ces clichés à fortes doses sont depuis toujours effectués la plupart du temps par les assistantes médicales sous la responsabilité du médecin compétent.

- Dans de nombreuses écoles, cela fait un certain temps que les bases des examens radiologiques à fortes doses sont enseignées, et ce afin de garantir une formation adaptée aux besoins de la pratique.

\section{Règlement d'apprentissage révisé en vigueur depuis le $1^{\text {er }}$ décembre 2003}

- Les raisons suivantes sont à la base du souhait d'élargissement des compétences octroyées en radiologie, exprimé par la FMH et les associations d'assistantes médicales:

a) Depuis toujours, des clichés à fortes doses sont exécutés dans quelque 4000 cabinets médicaux (dont presque 3000 appartenant à des médecins de premier recours) sous la responsabilité du médecin détenteur de la qualification technique.

b) L'assistante médicale chargée d'effectuer le cliché ne disposait jusqu'ici d'aucune possibilité de formation ou de perfectionnement pratique à ce sujet. 
c) Le véritable but visé par cette partie de la révision consistait à améliorer la radioprotection en intégrant les clichés radiologiques à fortes doses.

- Ont été nouvellement admis, les clichés radiologiques de la colonne vertébrale, du bassin, de l'abdomen et du crâne. La formation théorique est passée de 40 à 70 heures, mais la formation pratique comprend toujours 120 heures durant le cours d'introduction.

- Le catalogue des clichés radiologiques évalués lors de l'examen de fin d'apprentissage a été adapté en conséquence.

- Cette révision a été mise en vigueur par l'OFFT à la demande de la FMH et des associations d'assistantes médicales. Pour des raisons de temps, la procédure de consultation a été brève. La mise en vigueur du nouveau règlement d'apprentissage devait impérativement avoir lieu avant l'entrée en vigueur de la nouvelle loi sur la formation professionnelle ( $1^{\text {er }}$ janvier 2004). L'OFSP a été régulièrement informé de toutes les étapes.

\section{A la demande des radiologues, des techniciennes et techniciens diplômés en radiologie médicale (TRM) et de la Commission fédérale de protection contre les radiations et de surveillance de la radioactivité (CPR), I'OFSP bloque I'entrée en vigueur du règlement depuis début 2004}

Ci-après les principaux points litigieux ainsi que les contre-arguments avancés par la FMH:

- Les radiologues et les TRM craignent que les technicien-ne-s en radiologie soient remplacé-e-s par des assistantes médicales au bénéfice de compétences élargies en radiologie, dont les prestations sont moins chères. Cet argument ne semble guère valable étant donné que les radiologues peuvent d'ores et déjà engager des assistantes médicales et leur faire suivre un des cours homologués par l'OFSP et la CPR existant depuis 1998. En outre, les activités radiologiques des TRM englobent encore bien d'autres domaines (CT, IRM, US, examens avec produits de contraste, etc.).

- L'application de la formation pratique apportera son lot de difficultés.

Cela pose certainement un problème, étant donné qu'aujourd'hui encore la formation pratique pour la prise de clichés conven- tionnels n'est pas facile à dispenser. Il existe cependant des modèles qui permettraient de réaliser l'ensemble des clichés exigés durant les premières années d'activité professionnelle. Lors d'une conférence de conciliation, tant l'OFSP que les radiologues ont estimé qu'un tel compromis était applicable.

- La crainte d'une augmentation des clichés radiologiques à fortes doses est évoquée.

Cette crainte est infondée puisque l'indication est posée par le médecin qui doit disposer de la compétence radiologique nécessaire. Il est vrai cependant que l'exigence disproportionnée du nombre de clichés à examiner pourrait créer de fausses incitations amenant à l'extension de l'indication. C'est pourquoi nous nous opposons à l'instauration d'un nombre irréaliste de clichés obligatoires.

- Le nombre de clichés exigés pour obtenir une qualité suffisante en cas de radiographies du squelette axial, techniquement plus difficiles à réaliser, ne serait pas suffisant.

Même une formation pas tout à fait optimale vaut mieux que pas de formation du tout. On réalise déjà ce genre de clichés ... Et pour ce qui est des clichés traditionnels, il arrive souvent que les assistantes ne puissent pas non plus acquérir une routine suffisante lors de leur apprentissage.

- Seul un petit nombre d'assistantes médicales ont besoin de cette formation.

Comme mentionné plus haut, environ 4000 médecins sont détenteurs de la qualification technique et, parmi eux, quelques 3000 médecins de premier recours effectuent des radiographies dans leur cabinet.

Séance de conciliation à I'OFFT

du 13 juillet 2004 avec des représentants de I'OFFT, de I'OFSP, de la FMH (responsables des assistantes médicales), des associations d'assistantes médicales, des radiologues et des TRM

- L'élargissement de la formation théorique en radiologie, y compris l'intégration des clichés à fortes doses, est incontesté.

- Les radiologues et les TRM considèrent que la formation pratique n'est guère applicable et il a été impossible d'aboutir à un consensus.

- Il n'a pas non plus été possible de trouver un accord concernant les exigences pour un certificat correspondant. 
En août 2004, une proposition de compromis a été formulée

Celle-ci a été élaborée en collaboration entre la FMH et l'OFSP. La FMH s'est chargée de la rédiger et de la soumettre officiellement sous forme de proposition à l'OFSP le 9 septembre 2004. La voie semblait libre.

\section{Depuis le dépôt de la proposition le 9 septembre 2004, la décision revient à I'OFSP}

- Malgré des demandes répétées de notre part, l'OFSP n'a pris aucune décision contraignante jusqu'ici.

- Lors de la rencontre du 24 janvier 2005 entre les représentants de l'OFSP et les responsables décisionnels de la FMH, une nouvelle solution de compromis semblait possible.

- Lors de la rencontre annuelle des experts cantonaux en radiologie pour le domaine des assistantes médicales du 27 janvier 2005, un représentant de l'OFSP a assuré que la formation en examens radiologiques à fortes doses serait introduite comme prévue dans les écoles et qu'un compromis était en vue en ce qui concerne la formation pratique.

- Au début du mois de mars 2005, une rencontre au sommet s'est tenue entre le président de la FMH et le directeur de l'OFSP. Ce dernier a promis qu'une décision serait rapidement prise.

- A la mi-mars 2005, la CPR a refusé, en tant qu'organe consultatif de l'OFSP, d'octroyer la compétence élargie en radiologie aux assistantes médicales. La commission a pris cette décision après s'être réunie plusieurs fois. Dans l'argumentation de la CPR, la plupart des motifs que nous avions invoqués sont restés lettre morte (cf. point 3 ci-dessus).

- A la mi-août 2005, nous n'avons toujours pas reçu de réponse à la proposition soumise à l'OFSP en septembre 2004.

\section{Bilan d'une histoire insupportable}

A l'heure actuelle, donc un an et neuf mois après l'entrée en vigueur du nouveau règlement, la première volée d'assistantes médicales à former selon ce dernier, entame sa deuxième année d'apprentissage. Les écoles et les maîtres d'apprentissage attendent toujours la décision de l'OFSP pour enfin pouvoir appliquer les modifications dudit règlement.

La proposition négative de la CPR à l'intention de l'OFSP est incompréhensible et n'apporte aucune amélioration en ce qui concerne la radioprotection de la population étant donné qu'aucun cliché supplémentaire ne serait effectué. En outre, la part d'exposition de la population aux rayonnements de la radiologie diagnostique dans les cabinets de premier recours n'est que de 9,5\%, comme le montre une étude financée par l'OFSP et réalisée par l'institut de radiophysique du Prof. J. F. Valley - membre de la CPR - à Lausanne en l'an 2000.

Il est incompréhensible que l'OFSP se laisse à ce point freiner par une commission consultative et inadmissible qu'une question déposée voici un an, et discutée avec des spécialistes de cet office, soit restée sans réponse. 